\title{
Proton-conductivity-enhancing Ionic Liquid Consisting of Guanidine and Excess Trifluoromethanesulfonic Acid
}

Michiaki Kuroha, Hiroaki Gotoh, Muhammed Shah Miran, Tomohiro Yasuda, Masayoshi Watanabe, and Kazuhisa Sakakibara* Department of Advanced Materials Chemistry, Yokohama National University, 79-5 Tokiwadai, Hodogaya-ku, Yokohama, Kanagawa 240-8501

(E-mail: mozart@ynu.ac.jp)

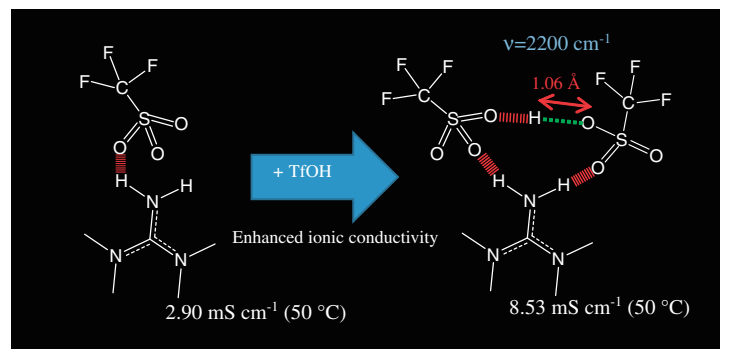

Proton-conductivity-enhancing ionic liquid has been prepared from 1,1,3,3-tetramethylguanidine (TMG) and excess trifluoromethanesulfonic acid (TfOH). Incorporation of hydrogen-bond system into the ionic liquids composed of guanidine and TfOH can embody enhanced proton conductivity by forming cluster structure. Fast mobile nature of conductive protons has been characterized by NMR, IR spectroscopy and B3LYP/6-31G* calculations.

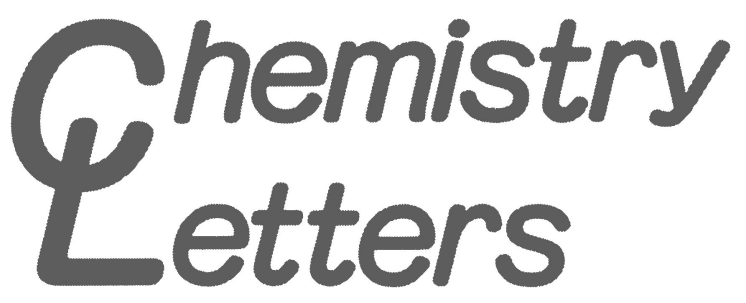

Vol.43 No.5 2014 p.649-651

CMLTAG

May 5, 2014

The Chemical Society of Japan 


\title{
Proton-conductivity-enhancing Ionic Liquid Consisting of Guanidine and Excess Trifluoromethanesulfonic Acid
}

\author{
Michiaki Kuroha, Hiroaki Gotoh, Muhammed Shah Miran, Tomohiro Yasuda, Masayoshi Watanabe, and Kazuhisa Sakakibara* \\ Department of Advanced Materials Chemistry, Yokohama National University, \\ 79-5 Tokiwadai, Hodogaya-ku, Yokohama, Kanagawa 240-8501
}

(E-mail: mozart@ynu.ac.jp)

Proton-conductivity-enhancing ionic liquid (IL) has been prepared from 1,1,3,3-tetramethylguanidine (TMG) and excess trifluoromethanesulfonic acid (TfOH). The fast mobile nature of conductive protons has been characterized by NMR, IR spectroscopy and B3LYP/6-31G* calculations.

Protic ionic liquid (PIL) such as diethylmethylammonium trifluoromethanesulfonate ([Dema][TfOH]) is considered to be a good electrolyte for nonhumidified intermediate temperature fuel cells. ${ }^{1,2}$ Essential properties of the electrolytes for fuel cells are higher proton conductivity, thermal stability, low vapor pressure, and facile fuel cell electrochemical reactions. ${ }^{3}$ Higher proton conductivity, by necessity, requires dissociation of the PIL due to the acid-base equilibrium (eq 1). However, thermally stable ILs need an equilibrium constant of formation high enough to prevent dissociation. Further, generation of the Brønsted base due to the release of $\mathrm{H}^{+}$for oxygen reduction reactions may result in the degradation of IL. ${ }^{3}$

Brønsted acid + Brønsted base $\rightleftarrows$ Ionic Liquid (IL)

In order to enhance proton conductivity of the PILs drastically, an inventive method to exert proton-hopping (Grotthuss-type $e^{4,5}$ characteristics of proton conduction) effectively in addition to the vehicle ${ }^{6}$ (diffusion control) is essential. The addition of excess base to PILs is one way to enhancing proton conductivity. ${ }^{3,7}$ To the contrary, our method is to use the guanidine base with an excess of TfOH leading to ample mobile protons under the circumstances of masking bare acidic protons by incorporating hydrogen-bond networks in the ILs. As guanidine $\left(\mathrm{p} K_{\mathrm{aH}}=13.6\right)$ is a superstrong base and has three basic sites, two basic sites remain even after the formation of thermally very stable PILs by the protonation of the imine nitrogen. Thermal stability of the guanidine-type ILs can be rationalized from the large $\Delta \mathrm{p} K_{\mathrm{a}}$ of the constituent amine and acid. ${ }^{8}$ If we can add fast mobile protons to these equimolar guanidine-type ILs through incorporation into the hydrogen-bond network, higher proton conductivity may be realized without dissociation of the PILs. In this paper, we report intriguing enhanced proton conductivity under excess acid (trifluoromethanesulfonic acid: $\mathrm{TfOH}$ ) conditions in the guanidine-type ILs such as [TMG][TfOH] (1:1.5) (TMG: 1,1,3,3-tetramethylguanidine, Chart 1) and their characteristic fast proton transferability which can be recognized from NMR, IR spectroscopy, and DFT calculations. As [TMG][TfOH] IL is symmetric, $\mathrm{N}-\mathrm{H}$ protons in ${ }^{1} \mathrm{HNMR}$ and IR spectra can be clearly characterized.

The protic ILs were prepared by adding TfOH dropwise into neat guanidine in a three-necked flask at $-30^{\circ} \mathrm{C}$ under $\mathrm{N}_{2}$ atmosphere. ${ }^{1} \mathrm{H}$ - and ${ }^{15} \mathrm{~N}$ NMR measurements were carried out with a JEOL ECX-400 spectrometer by using a $5 \mathrm{~mm} \phi$ double<smiles>CN(C)C(=[NH2+])N(C)C</smiles>

1.1.3.3-tetramethylguanidine (TMG)

\section{Chart 1.}

Table 1. Characteristic ${ }^{1} \mathrm{H}$ NMR properties of mobile $\mathrm{N}-\mathrm{H}$ protons ( $\delta$, peak shape, and peak integration values) in the ILs formed between Brønsted bases and $\mathrm{TfOH}$

\begin{tabular}{clcccc}
\hline Entry & Compound & $\begin{array}{c}\text { TfOH ratio } \\
\text { to base }\end{array}$ & $\begin{array}{c}\mathrm{N}-\mathrm{H} \\
(\delta)\end{array}$ & $\begin{array}{c}\text { Integral } \\
\text { value/H }\end{array}$ & $\begin{array}{c}\text { Half-width } \\
/ \mathrm{Hz}\end{array}$ \\
\hline 1 & {$[\mathrm{Dema}][\mathrm{TfOH}]$} & 1.0 & 7.31 & 0.99 & 32.1 \\
2 & {$[\mathrm{Dema}][\mathrm{TfOH}]$} & 2.0 & 6.65 & 0.95 & 53.7 \\
3 & {$[\mathrm{DBU}][\mathrm{TfOH}]$} & 1.0 & 7.94 & 1.00 & 4.7 \\
4 & {$[\mathrm{DBU}][\mathrm{TfOH}]$} & 2.0 & 7.91 & 0.97 & 19.23 \\
5 & {$[\mathrm{TMG}][\mathrm{TfOH}]$} & 1.0 & 6.24 & 1.97 & 12.26 \\
6 & {$[\mathrm{TMG}][\mathrm{TfOH}]$} & 1.5 & 6.53 & 1.13 & 552.4 \\
7 & {$[\mathrm{TMG}][\mathrm{TfOH}]$} & 2.0 & 6.91 & 0.78 & 1321 \\
8 & {$[\mathrm{TMG}][\mathrm{TfOH}]$} & 3.0 & 9.70 & 2.14 & 324.1 \\
\hline
\end{tabular}

sample tube (inner: PIL: outer: DMSO- $d_{6}$ with TMS). FT-IR spectra were recorded on a Thermo Fischer SCIENTIFIC NICOLET iS10. Ionic conductivities were determined employing the complex impedance method with a potentiostat (Autolab, PGSTAT30). Differential scanning calorimetry (DSC) was carried out on a Seiko Instruments DSC 220C, and thermogravimetric analysis (TGA) was carried out using Seiko Instruments TG-DTA $6200 \mathrm{C}$ both under a nitrogen atmosphere.

${ }^{1} \mathrm{HNMR}$ data of the mobile N-H protons in PILs ([Dema]$[\mathrm{TfOH}],{ }^{2}$ [DBU][TfOH $]^{9}$ (DBU: 1,8-diazabicyclo[5.4.0]undec7-ene), and $[\mathrm{TMG}][\mathrm{TfOH}])$ are summarized in Table 1 . The chemical shift values $(\delta)$ of the $\mathrm{N}-\mathrm{H}$ are located at the intermediate region between acidic $\mathrm{TfOH}(\delta=9.93)$ and its counterpart base ( $\delta=5.12$ for TMG). Peak shape of the standard PIL composed of 1:1 acid and base is sharp with the signal integral intensity corresponding to the actual amount $(\mathrm{H})$ of the equimolar ILs. As expected, this $\mathrm{N}-\mathrm{H}$ proton was able to exchange smoothly with mobile $\mathrm{OH}$ proton, which could be ascertained from $\mathrm{D}_{2} \mathrm{O}$ exchange.

It is worthwhile to note that peak shape becomes broader as acid molar ratio increases over the equivalent point for all ILs suggesting faster intermolecular proton transfer occurs. Representative ${ }^{1} \mathrm{H}$ NMR spectra are shown in Supporting Information. This $\mathrm{N}-\mathrm{H}$ peak broadening feature is especially remarkable in 


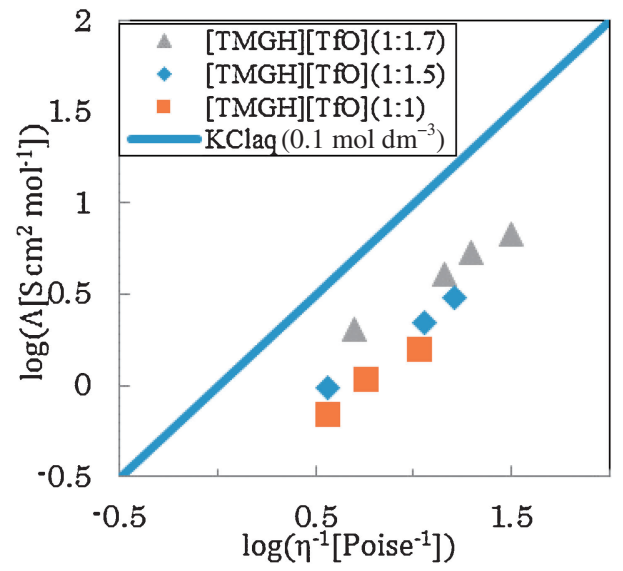

Figure 1. Walden plot of the $[\mathrm{TMG}][\mathrm{TfOH}]$ ILs with varying molar ratio of $[\mathrm{TfOH}]$.

[TMG][TfOH] IL. In the case of TfOH-excess IL such as [TMG][TfOH] (1:1.5), excess acidic protons may be able to interact with the remaining basic sites in the TMG, and faster proton transfer seems to occur without emergence of bare protons intrinsic to excess $\mathrm{TfOH}$. In addition to peak broadening, decrease of $\mathrm{N}-\mathrm{H}$ proton intensities (integrated values) was observed for the acid excess ILs. In the case of monitoring faster movable $\mathrm{N}-\mathrm{H}$ protons properly in the acid-excess ILs, ${ }^{1} \mathrm{HNMR}$ time scale is too slow to respond to fast dynamic $\mathrm{N}-\mathrm{H}$ proton transfer promptly. Thus, $\mathrm{N}-\mathrm{H}$ proton signal intensity shows smaller values than those of actual $\mathrm{N}-\mathrm{H}$ protons in the acid-excess PILs. The fade away of the spin-spin coupling between ${ }^{1} \mathrm{H}$ and ${ }^{15} \mathrm{~N}$ nuclei $\left({ }^{1} J_{\mathrm{N}-\mathrm{H}}=147 \mathrm{~Hz}\right)$ of the iminium nitrogen $(\delta=77.6)$ observed in the [TMG][TfOH] $(1: 1)$ by adding excess TfOH to the equimolar IL also rationalizes faster $\mathrm{N}-\mathrm{H}$ proton exchange in the acid-excess ILs ([TMG][TfOH] $(1: 1.5))$. In order to be sure of the faster proton hopping, ionic conductivities $\left(\sigma / \mathrm{S} \mathrm{cm}^{-1}\right)$ of $[\mathrm{TMG}][\mathrm{TfOH}]$ with varying [TMG] to [TfOH] ratio from 1:1 through $1: 2$ have been measured. On the basis of the measured ionic conductivities, a Walden plot $\left(\log \Lambda\right.$ vs. $\left.\log \eta^{-1}\right)$ was drawn in Figure 1. Ionic conductivity increases proportionally depending on the amount of excess $\mathrm{TfOH}$ and approaches closer to the standard aqueous $\mathrm{KC} 1$ solution $\left(0.1 \mathrm{~mol} \mathrm{dm}^{-3}\right)$ (blue line; $\sigma / \mathrm{mS} \mathrm{cm}^{-1}$ at $T=$ $50{ }^{\circ} \mathrm{C}$ : [TMG][TfOH] (1:1), 2.89; [TMG][TfOH] (1:1.5), 5.72; [TMG][TfOH] $(1: 1.7), 8.53)$. The observed increase of the ionic conductivities may be ascribed to (1) larger number of the proton carriers, (2) decrease of the viscosity of IL leading to faster mobility, or (3) proton hopping. At present, definite evidence supporting proton hopping (Grotthuss mechanism) which can be clarified from PGSE $\mathrm{NMR}^{3}$ measurements and ionic conductivity experiments by Kreuer ${ }^{10}$ are not available.

It is intriguing that exchangeable protons incorporated into the hydrogen-bond network in the TfOH-excess ILs such as [TMG][TfOH] (1:1.5) can enhance proton conductivity without emerging acidic bare protons.

In order to further investigate the faster $\mathrm{N}-\mathrm{H}$ proton-transfer behavior in the [TMG][TfOH] IL, IR spectroscopic analyses have been carried out by taking advantage of the faster time scale to monitor dynamic N-H proton movement. Typical IR spectra of $[\mathrm{TMG}][\mathrm{TfOH}]$ with varying amount of $\mathrm{TfOH}$ are

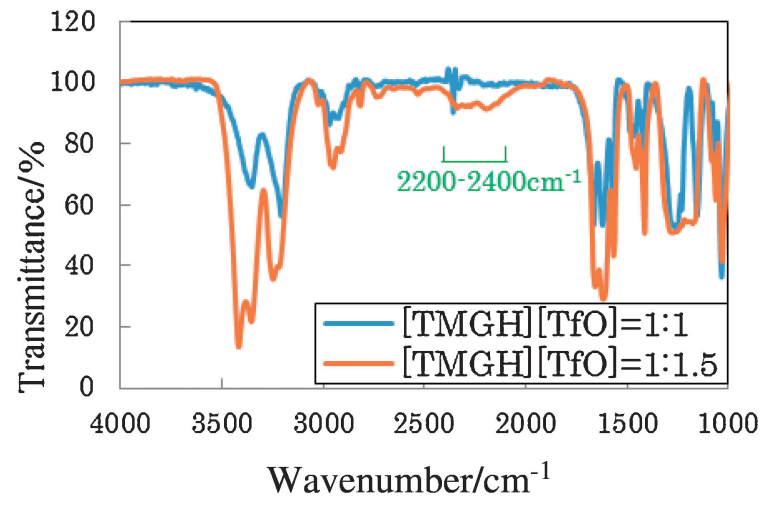

Figure 2. IR spectra of $[\mathrm{TMG}][\mathrm{TfOH}]$ ILs with varying molar ratio of $[\mathrm{TMG}]:[\mathrm{TfOH}]$.

shown in Figure 2. While equimolar [TMG][TfOH] (1:1) IL shows characteristic $\mathrm{NH}_{2}$ antisymmetric $\left(3353 \mathrm{~cm}^{-1}\right)$ and symmetric $\left(3208 \mathrm{~cm}^{-1}\right)$ stretching vibration peaks, excess TfOHcontaining $[\mathrm{TMG}][\mathrm{TfOH}](1: 1.5)$ exerts further splitting of $\mathrm{N}-\mathrm{H}$ vibrational peaks due to the increase of mobile $\mathrm{N}-\mathrm{H}$ protons enabling interaction with the guanidine base to give rise to variant conformations with regard to the iminium moiety. However, O-H stretching vibration which may appear due to excess TfOH $\left(3040 \mathrm{~cm}^{-1}\right.$ broad peak due to association in liquid) ${ }^{11}$ could not be observed. In addition, these $\mathrm{N}-\mathrm{H}$ stretching bands disappeared and moved to lower frequency region by the substitution of $\mathrm{N}-\mathrm{H}$ proton with deuterium (D) according to the physical equation $\left(\nu \propto \mu^{-1 / 2}\right)$ correlating frequency $(\nu)$ with the reduced mass $(\mu)$ of forming the bond $(\mathrm{N}-\mathrm{H}$ or $\mathrm{N}-\mathrm{D})$. After $\mathrm{H}-\mathrm{D}$ exchange experiments, $\mathrm{NH}_{2}$ antisymmetric and symmetric stretching bands shifted to 2942 and $2844 \mathrm{~cm}^{-1}$, respectively. By adding excess $\mathrm{TfOH}$, the intensity of these transferable $\mathrm{N}-\mathrm{H}$ peaks increases due to the newly-formed hydrogen-bond network. Furthermore, it is intriguing that a new broad band appears increasingly around 2200 through $2400 \mathrm{~cm}^{-1}$ dependent on the amount of excess $\mathrm{TfOH}$. This characteristic broader band moved to further lower frequency (ca. $2000 \mathrm{~cm}^{-1}$ ) by deuterium exchange (see Supporting Information). It should be noticed that this lower frequency shift was not accounted for by the $\left(v \propto \mu^{-1 / 2}\right)$ equation in this case, however. What kind of vibrational mode is inherent in this broad IR band (2200-2400 $\left.\mathrm{cm}^{-1}\right)$ ? It is plausible to assume that this broad peak comes from hydrogen-bond formation where excess protons originating from $\mathrm{TfOH}$ interact with the guanidine base although frequency numbers around 2200$2400 \mathrm{~cm}^{-1}$ are too low for the $\mathrm{O}-\mathrm{H}$ hydrogen bond. However, anomalously lower shifted $\mathrm{O}-\mathrm{H}$ hydrogen bonded peaks have been reported recently to appear (around $2500 \mathrm{~cm}^{-1}$ ) in a cluster system consisting of proline and coordinated chloride ion from infrared photodissociation experiments and computational study. ${ }^{12}$

To be sure of the vibrational mode of this lower-frequencyshifted broad peak, theoretical calculation has been carried out at the B3LYP/6-31G* level. Considering various plausible configurations of the cluster structures with [TMG][TfOH] (1:2) composition, geometries were optimized and frequency analyses were carried out. The most stable cluster structure is shown in Figure 3 where heavy atoms $(\mathrm{O}$ or $\mathrm{N})$ which comprise a 


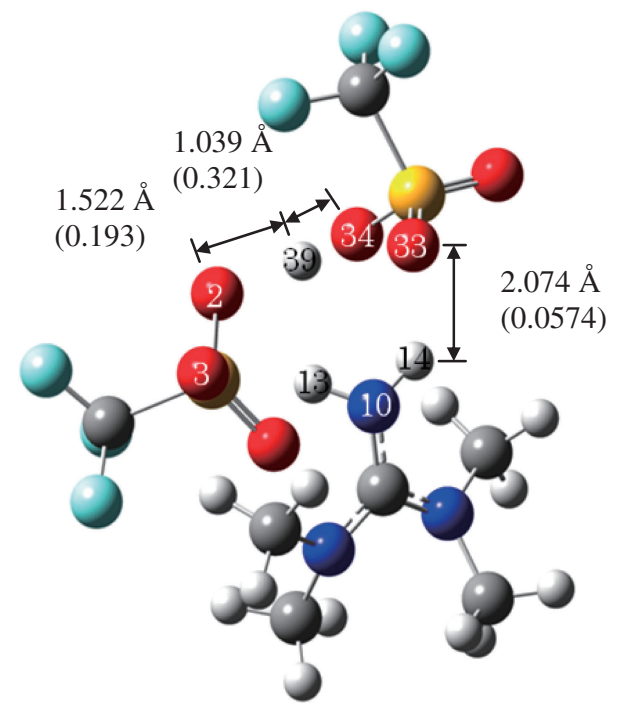

Figure 3. The optimized cluster structure of $[\mathrm{TMG}][\mathrm{TfOH}]$ (1:2) rationalizing IR peaks inherent to fast moving protons contributing to higher proton conductivity. Interatomic distances and related bond orders (in the parentheses) are shown.

hydrogen-bond system (X-H...Y) are located at positions compatible with ordinary intermolecular $\mathrm{O}-\mathrm{H} \ldots \mathrm{O}$ or $\mathrm{N}-\mathrm{H} \cdots \mathrm{O}$ hydrogen bonds. The distances of the $\mathrm{O}(2) \cdots \mathrm{O}(34)(2.56 \AA)$ and $\mathrm{N}(10) \ldots \mathrm{O}(33)(2.92 \AA)$ found in the optimized cluster structure are adaptable ones found as the average heavy-atom distances of the hydrogen-bond system compiled in the Cambridge Structural Database (CSD). ${ }^{13}$ Movable proton $\mathrm{H}(39)$ inherent to excess $\mathrm{TfOH}$ is coordinated around iminium nitrogen $\mathrm{N}(10)$ being far apart, about $4 \AA$. The distance between this movable proton $\mathrm{H}(39)$ and the nearest sulfonyl oxygen $\mathrm{O}(34)$ is $1.039 \AA$ indicating considerable lengthening of the $\mathrm{O}-\mathrm{H}$ bond. ${ }^{14}$

In order to be sure of the cluster structure as shown in Figure 3, FAB (fast atom bombardment) mass experiments have been carried out in negative ion mode. In the FAB mass spectra of the [TMG][TfOH] $(1: 1.7) \mathrm{IL} m / z: 414$ peak corresponding to $\left[2 \mathrm{TfO}^{-}+\mathrm{TMGH}^{+}\right]$was observed clearly. This FAB mass result supports the existence of the cluster structure where mobile protons are incorporated into the hydrogen-bond network and are contributing the enhanced proton conductivity.

Mobile protons providing enhanced proton conductivity in the TfOH-excess [TMG][TfOH] characterized by IR, NMR, FAB-mass experiments and DFT calculations are surely those incorporated into the hydrogen-bond network formed in the cluster structure as shown in Figure 3 and may be available for smooth proton hopping because a rapid association-dissociation hydrogen-bond system may be able to embody the cluster structure. ${ }^{15}$ However, interaction energy acquired by the hydrogen-bond formation is not very large because interatomic distances between mobile protons and coordinated hydrogen bond donor atoms $(\mathrm{O}$ or $\mathrm{N})$ are too long. Therefore, the cluster structure shown in Figure 3 has been deduced to be degradable over $150^{\circ} \mathrm{C}$ by taking into account the results of TGA and DSC experiments. ${ }^{16}$ Optimum compatibility between high proton conductivity and thermal stability is requisite to make superior ILs for the electrolytes for fuel cells.

Based on the [TMG][TfOH] $(1: 1)$ IL, adding a little more excess $\mathrm{TfOH}$ leads to producing higher proton conductivity and lower viscosity ILs with rather high thermal stability. Fast mobile nature and extremely lower-frequency hydrogen-bond formation inherent to TfOH-excess [TMG][TfOH] ILs have been characterized by various spectroscopic (NMR, IR, and FAB-MS) experiments and theoretical (B3LYP/6-31G*) calculations. The origins of fast mobile protons are hydrogen-bonded protons which are incorporated into cluster structure consisting of guanidine base and $\mathrm{TfOH}$.

\section{References and Notes}

1 A. Noda, M. A. B. H. Susan, K. Kudo, S. Mitsushima, K. Hayamizu, M. Watanabe, J. Phys. Chem. B 2003, 107, 4024.

2 H. Nakamoto, M. Watanabe, Chem. Commun. 2007, 2539.

3 S.-Y. Lee, A. Ogawa, M. Kanno, H. Nakamoto, T. Yasuda, M. Watanabe, J. Am. Chem. Soc. 2010, 132, 9764.

4 C. J. T. van Grotthuss, Ann. Chim. 1806, 58, 54.

5 R. Vuilleumier, D. Borgis, Nat. Chem. 2012, 4, 432.

6 K.-D. Kreuer, A. Rebenau, W. Weppner, Angew. Chem., Int. Ed. Engl. 1982, 21, 208.

7 J. A. Bautista-Martinez, L. Tang, J.-P. Belieres, R. Zeller, C. A. Angell, C. Friesen, J. Phys. Chem. C 2009, 113, 12586.

8 M. S. Miran, H. Kinoshita, T. Yasuda, M. A. B. H. Susan, M. Watanabe, Phys. Chem. Chem. Phys. 2012, 14, 5178.

9 M. S. Miran, H. Kinoshita, T. Yasuda, M. A. B. H. Susan, M. Watanabe, Chem. Commun. 2011, 47, 12676.

10 K.-D. Kreuer, S. J. Paddison, E. Spohr, M. Schuster, Chem. Rev. 2004, 104, 4637.

11 E. L. Varetti, Spectrochim. Acta, Part A 1988, 44, 733.

12 J. Schmidt, S. R. Kass, J. Phys. Chem. A 2013, 117, 4863.

13 http://www.ccdc.cam.ac.uk/Pages/Home.aspx, CSD (Cambridge Structural Database).

14 Considering the natural bond length of O-H (0.947 $)$, calculated O-H length $(1.039 \AA)$ is assumed to be too long as a normal length for a hydrogen-bonded system. Bond order of this $\mathrm{O}-\mathrm{H}$ bond was too small $(0.321)$ to be recognized as a normal bond while the bond order of the $\mathrm{N}-\mathrm{H}$ bonds in [TMG] [TfOH] (1:1) which showed two $\mathrm{N}-\mathrm{H}$ peaks clearly around $3400 \mathrm{~cm}^{-1}$ in Figure 2 are around 0.5 .

15 L. Vilčiauskas, M. E. Tuckerman, G. Bester, S. J. Paddison, K.-D. Kreuer, Nat. Chem. 2012, 4, 461.

16 TGA and DSC charts of [TMG][TfOH] ILs, and representative ${ }^{1} \mathrm{HNMR}$ and IR spectra are shown in Supporting Information. Supporting Information is available electronically on the CSJ-Journal Web site, http://www.csj.jp/ journals/chem-lett/index.html. 